\title{
Mehr Möglichkeiten und attraktive All-in-Sets
}

iSy ${ }^{\circledR}$ ist das preiswerte Qualitätssystem von CAMLOG. Es ist schlank, flexibel und überzeugt in der Praxis mit einfacher Handhabung, einem effizientem Workflow und sehr günstigen Preisen. Zum 1. Juli 2015 hat CAMLOG das Implantatsystem erweitert und kommt damit dem Wunsch vieler Anwender nach, die die Implantate in noch mehr Fällen einsetzen möchten.

Neu ins Produktprogramm aufgenommen wurden 7,3 mm kurze Implantate. Sie sind für Fälle mit eingeschränktem Knochenangebot geeignet und erweitern das Indikationsspektrum des Systems. Ebenfalls neu erhältlich sind direkt im Implantat verschraubbare Esthomic Gingivaformer, die Möglichkeit der Abformung auf Implantatniveau durch offene und geschlossene Abformpfosten sowie verschiedene

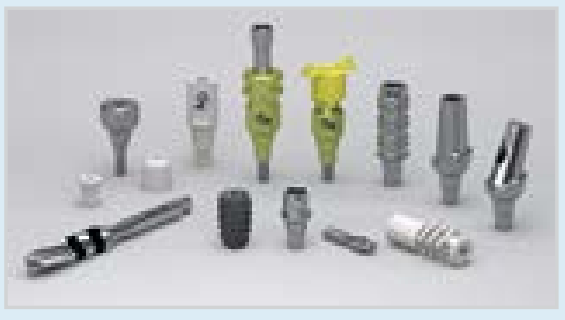

prothetische Komponenten und Instrumente. Die neuen Esthomic-Abutments erlauben ästhetische zementierte Rekonstruktionen. Ihr Austrittsprofil ist formkongruent mit dem der Esthomic Gingivaformer und Abformpfosten offener und geschlossener Löffel. Gleichzeitig wurde vom Hersteller die Implantatbasis auch für definitive Versorgungen freigegeben. Durch diese Erweiterungen stehen dem Behandlungsteam nun noch mehr Optionen offen - und dies unter voller Beibehal- tung des ursprünglichen iSy-Konzepts und dessen Vorteile an Einfachheit und Effizienz. Das Konzept des iSy-Implantatsystems beruht auf den All-in-ImplantatSets, die nicht nur 1 bzw. 4 Implantate beinhalten, sondern wertvolle Zusatzteile. Das sind die im Implantat vormontierten Implantatbasen, ein Einpatienten-Formbohrer, Gingivaformer und Multifunktionskappen zum Scannen, Abformen und provisorisch Versorgen. Gingivaformer und Multifunktionskappen bestehen aus PEEK und werden einfach auf die Implantatbasis aufgesteckt. Weitere Informationen zum iSy-Implantatsystem unter 07044 9445-100 oder auf www.isy-implant.de.

Nach einer Pressemitteilung der CAMLOG Vertriebs GmbH, Wimsheim Internet: www.camlog.de 